\title{
TWO PROOFS OF THE STABLE ADAMS CONJECTURE
}

\author{
BY ERIC M. FRIEDLANDER ${ }^{1}$ AND R. M. SEYMOUR \\ Communicated by R. James Milgram, March 14, 1977
}

Let $p$ and $q$ be distinct primes. The complex Adams conjecture establishes a homotopy commutative diagram

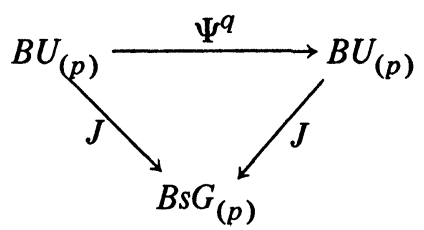

where $J$ is the complex $J$-homomorphism and $-(p)$ denotes localization at $p$. Both $J$ and $\Psi^{q}$ are infinite loop maps, and it is natural to ask whether this result is infinitely deloopable; that is, whether $J \Psi^{q}=J$ as infinite loop maps. This is the Stable Adams Conjecture.

We announce here two independent proofs of this conjecture. Details will appear in [2] and [6].

METHOD 1. Our proof is based upon a "geometric" criterion for pairs of maps into the spectrum $(\mathrm{BsG})^{\wedge} \cong(\mathrm{BsG})_{(p)}$ to be homotopic, where ( $)^{\wedge}$ denotes the Bousfield-Kan $\mathbf{Z} / p$-completion functor. We exploit the "galois symmetry" of $(\mathrm{kU})^{\wedge}[8]$ to show that $J^{\wedge}, J^{\wedge} \circ\left(\Psi^{q}\right)^{\wedge}$ satisfy this criterion.

We impose a Quillen closed model category structure on Segal's $\Gamma$-spaces [3], whose weak equivalences are level-wise weak equivalences of spaces. For any "suitably oriented, pointed C. W.-like space" $X$ (e.g., $X$ any pointed C. W. complex with no orientation specified), we obtain a $\Gamma$-space $B s G_{X}$ arising from distinguished homotopy equivalences of iterated smash products of $X$ with itself. There is a natural functor

$$
\Phi: \text { Ho } \Gamma \text {-spaces } \rightarrow \text { HoSpectra }
$$

sending $B s G_{s^{2}}$ to $\Phi\left(B s G_{S^{2}}\right)=\mathbf{B s G}$.

Our basic representability theorem is a description of the functor

$$
\text { Hom }_{\text {Ног-spaces }}\left(, B s G_{X}\right)
$$

as being isomorphic to the functor $s X()$ of "oriented $X$-structures" over a variable $\Gamma$-space as base. For sufficiently nice $X\left(\right.$ e.g., $\left.X=S^{2}\right), \operatorname{Hom}_{\mathrm{Ho} \text {-spaces }}\left(,\left(B s G_{X}\right)^{\wedge}\right)$ is isomorphic to $\mathrm{Z} / p s X($ ) (the theory of "oriented, $\mathrm{Z} / p$-completed $X$-structures"). The critical property of these $X$-structures is that they admit a functorial principal-

AMS (MOS) subject classifications (1970). Primary 55B1 5, 55 F50.

1 Partially supported by the N.S.F.

Copyright @ 1977, American Mathematical Society 
ization such that the principalization of the universal oriented $X$-structure $\pi_{X}$ : $B\left(s G_{X}, X\right) \rightarrow B s G_{X}$ is weakly contractible.

The complex $J$-homomorphism $J: \mathbf{k U} \rightarrow \mathbf{B s G}$ is of the form $\Phi(\mathrm{J}: B G L \rightarrow$ $\left.B s G_{S^{2}}\right)$. Moreover, the oriented $S^{2}$-structure $\pi: B(G L, S) \rightarrow B G L$ induced by $J$ is algebraic in the sense that it arises from a pair of $\Gamma$-objects of simplicial complex algebraic varieties, $\pi_{C}: B\left(G L_{C}, S_{C}\right) \rightarrow B G L_{C}$. The $\mathbf{Z} / p$-completion of the rigid etale homotopy type of $\pi_{C}$,

$$
\pi_{\text {rêt }}:\left(\left(B\left(G L_{C}, S_{C}\right)\right)_{\text {ret }}\right)^{\wedge} \rightarrow\left(\left(B G L_{C}\right)_{\text {ret }}\right)^{\wedge}
$$

is $\mathbf{Z} / p$-completed $S^{2}$-structure equivalent to $\pi^{\wedge}$.

A (discontinuous!) automorphism $\phi: C \rightarrow C$ determines a map of pairs $\left(\tilde{\phi}_{\text {ret }}, \phi_{\text {ret }}^{\hat{n}}\right): \pi_{\text {ret }} \rightarrow \pi_{\text {ret }}$, and thus a map $\left(\tilde{\phi}^{\wedge}, \phi^{\wedge}\right): \pi^{\wedge} \rightarrow \pi^{\wedge}$. We conclude that $\left(\phi^{\wedge}\right)^{*}\left(\pi^{\wedge}\right)$ is equivalent to $\pi^{\wedge}$, so that $J^{\wedge} \circ \phi^{\wedge}$ is homotopic to $J^{\wedge}$. In particular, if $\phi: C \rightarrow C$ is any extension of the $q$ th root map on the Witt vectors of the algebraic closure of the prime field $F_{q}$, then $\Phi\left(\phi^{\wedge}\right)=\left(\Psi^{q}\right)^{\wedge}$. Thus $J^{\wedge}$ 。 $\left(\Psi^{p}\right)^{\wedge}$ is homotopic to $J^{\wedge}$ as maps of spectra.

The same proof, with notational changes, applies to prove the quaternionic analogue of the Stable Adams Conjecture. The proof fails in the real case because the algebraic model for the orthogonal groups acts algebraically via the complexification of the usual action (i.e., $O_{n, C}$ acts on complex affine $n$-space and thus on $S^{2 n}$ ).

METHOD 2. Let $p$ and $q$ be distinct primes. We construct a category with "sum", $A d_{q}$, which by $[3, \S 2]$, defines a cohomology theory, $k_{A d_{q}}^{*}$. Further, if $V$ is the category of finite dimensional complex vector spaces and isomorphisms, under direct sum, so that $k_{v}^{*}$ is complex connective $K$-theory, there is a sum preserving forgetful functor $j: A d_{q} \rightarrow V$. Thus, we have an induced map of theories, $k_{j}^{*}: k_{A d_{q}}^{*} \rightarrow k_{v}^{*}$. The main step in our proof is

THEOREM (i) On localizing at $p, k_{j}^{*}$ becomes an isomorphism.

(ii) There is a sum preserving autofunctor $\sigma$ of $A d_{q}$ such that, under the isomorphism of (i), the p-localization of $k_{\sigma}^{*}$ corresponds to $\Psi^{q}$, the Adams operation of p-localized connective $K$-theory.

The theorem is proved in [5]. Here is the definition of $A d_{q} \cdot \operatorname{Ob}\left(A d_{q}\right)=$ $\mathrm{II}_{n \geqslant 0} K_{n}$, where $K_{n}$ is the space of "proper" (in the sense of [7, Definition 4.2]) $\mathbf{Z} / q$ isomorphisms over $\mathbf{Z}$,

$$
\theta: \mathrm{n}^{\otimes q} \rightarrow \sigma^{*}(\mathrm{n}) \oplus \mathrm{n}^{\prime} \otimes N
$$

where $\mathbf{n}=\mathbf{Z} \times \mathbf{C}^{n}, \mathbf{n}^{\prime}=\mathbf{Z} \times \operatorname{Hom}_{\mathbf{Z} / q}\left(V,\left(\mathbf{C}^{n}\right)^{\otimes q}\right), V$ being a 1-dimensional primitive representation of $\mathbf{Z} / q, N$ is the regular representation of $\mathbf{Z} / q$, and $\sigma: \mathbf{Z} \rightarrow$ $\mathbf{Z}$ sends $r$ to $r+1$. A morphism, $\alpha: \theta \rightarrow \phi$, in $A d_{q}$ is an isomorphism over $\mathbf{Z}$, $\alpha: \mathbf{n} \rightarrow \mathbf{n}$, such that $\left(\sigma^{*}(\alpha) \oplus \alpha^{\prime} \otimes N\right) \circ \theta=\phi^{\circ} \alpha^{\otimes q}$, where $\alpha^{\prime}=\operatorname{Hom}_{\mathbf{z} / q}\left(V, \alpha^{\otimes q}\right)$. The sum on $A d_{q}$ is defined as in $[4, \S 1]$. 
Define $\sigma: A d_{q} \rightarrow A d_{q}$ on objects by $\sigma(\theta)_{r}=\theta_{r+1}$, where $\theta_{r}=\theta \mid\{r\} \times$ $\left(\mathbf{C}^{n}\right)^{\otimes q}$, and on morphisms by $\sigma(\alpha)_{r}=\alpha_{r+1}$, where $\alpha_{r}=\alpha \mid\{r\} \times C^{n}$. Define $j: A d_{q} \rightarrow U$ by $j(\theta)=n$ for $\theta \in K_{n}$, and $j(\alpha)=\alpha_{0}$ for $\alpha \in \operatorname{Mor}\left(A d_{q}\right)$.

Now let $S_{d}$ be the category whose object space is $\mathbf{N}$ and whose morphisms, $\alpha: \mathbf{n} \rightarrow \mathbf{n}$, are proper homotopy equivalences of $\mathbf{R}^{n}$ of degree a power of $d$. The complex $J$-homomorphism is induced by the functor $J: V \rightarrow S_{1}\left(V \mapsto \operatorname{dim}_{R} V\right.$ on objects).

Let $S_{(p)}$ be the category with object space $\mathbf{N}$ and morphisms the space of homotopy equivalences of (standardized) $p$-local spheres (in the sense of [1]). For $(d, p) \equiv 1$, there is a sum preserving $p$-localization functor, $l: S_{d} \rightarrow S_{(p)}$. The $p$-localized complex $J$-homomorphism is recoverable from $l J: V \rightarrow S_{(p)}$.

Consider $J j: A d_{q} \rightarrow S_{1}$. We have $J j(\theta)=J j \sigma(\theta)$ for $\theta \in O b\left(A d_{q}\right)$, and $J j(\alpha)=\alpha_{0}, J j \sigma(\alpha)=\alpha_{1}$ for $\alpha \in \operatorname{Mor}\left(A d_{q}\right)$. Further, there is a commutative diagram

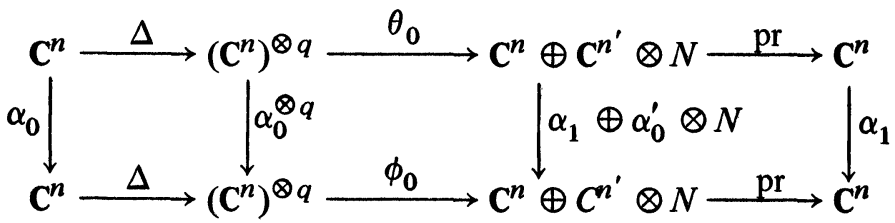

where $\mathrm{pr}$ is the projection, and $\Delta$ is the diagonal, $\Delta(v)=v^{\otimes q}$. Thus, setting $h_{\theta}=\operatorname{pr} \circ \theta_{0} \circ \Delta, h=\left\{h_{\theta}\right\}$ defines a natural transformation between $J j$ and $J j \sigma$ in $S_{q}$. Passing to $S_{(p)}, l h: l J j \rightarrow l J j \sigma$ is a natural transformation which is easily seen to preserve sums. This implies that $k_{l j j}^{*}=k_{l j j \sigma}^{*}: k_{A d_{q}}^{*} \rightarrow k_{S(p)}^{*}$. The conjecture is now proved by applying the above theorem.

\section{REFERENCES}

1. A. K. Bousfield and D. M. Kan, Homotopy limits, completions, and localizations, Lecture Notes in Math., vol. 304, Springer-Verlag, Berlin and New York, 1972.

2. E. M. Friedlander, Stable Adams conjecture via representability theorems for $\Gamma$. spaces (to appear).

3. G. Segal, Categories and cohomology theories, Topology 13 (1974), 293-312. MR 50 \#5782.

4. $\bullet$ R. M. Seymour, Vector bundles invariant under the Adams operations, Quart. J. Math. Oxford Ser. (2) 25 (1974), 395-414.

5. - A local representation of $\Psi^{q}$ (to appear).

6. - The infinite loop Adams conjecture (to appear). 269-302.

7. V. Snaith, The complex J-homomorphism, Proc. London Math. Soc. (3) 34 (1977),

8. D. Sullivan, Genetics of homotopy theory and the Adams conjecture, Ann. of Math. (2) 100 (1974), 1-79.

DEPARTMENT OF MATHEMATICS, NORTHWESTERN UNIVERSITY, EVANSTON, ILLINOIS 60201

DEPARTMENT OF MATHEMATICS, NORTHWESTERN UNIVERSITY, EVANSTON, ILLINOIS 60201 (Current address of E. M. Friedlander)

Current address (R. M. Seymour): Department of Mathematics, University College London, London, England 